APS

physics

This is the accepted manuscript made available via CHORUS. The article has been published as:

\title{
Readout of singlet-triplet qubits at large magnetic field gradients
}

Lucas A. Orona, John M. Nichol, Shannon P. Harvey, Charlotte G. L. Bøttcher, Saeed Fallahi, Geoffrey C. Gardner, Michael J. Manfra, and Amir Yacoby

Phys. Rev. B 98, 125404 - Published 5 September 2018 DOI: 10.1103/PhysRevB.98.125404 


\title{
Readout Of Singlet-Triplet Qubits At Large Magnetic Field Gradients
}

\author{
Lucas A. Orona, ${ }^{1}$ John M. Nichol, ${ }^{1,2}$ Shannon P. Harvey ${ }^{1}{ }^{2}$ Charlotte G. L. Bøttcher,${ }^{1}$ \\ Saeed Fallahi, ${ }^{3}$ Geoffrey C. Gardner, ${ }^{3}$ Michael J. Manfra, ${ }^{3,4,5,6}$ and Amir Yacoby ${ }^{1}$ \\ ${ }^{1}$ Department of Physics, Harvard University, Cambridge, MA 02138, USA \\ ${ }^{2}$ Department of Physics and Astronomy, University of Rochester, Rochester, NY 14627, USA \\ ${ }^{3}$ Department of Physics and Astronomy, Purdue University, West Lafayette, IN 47907, USA \\ ${ }^{4}$ School of Materials Engineering, Purdue University, West Lafayette, IN 47907, USA \\ ${ }^{5}$ Birck Nanotechnology Center, Purdue University, West Lafayette, IN 47907, USA \\ ${ }^{6}$ School of Electrical and Computer Engineering, \\ Purdue University, West Lafayette, IN 4790\%, USA
}

(Dated: June 15, 2018)

\begin{abstract}
Visibility of singlet-triplet qubit readout is reduced to almost zero in large magnetic field gradients due to relaxation processes. Here we present a new readout technique that is robust against relaxation and allows for measurement when previously studied methods fail. This technique maps the qubit onto spin states that are immune to relaxation using a spin dependent electron tunneling process between the qubit and the lead. We probe this readout's performance as a function of magnetic field gradient and applied magnetic field, and optimize the pulse applied to the qubit through experiment and simulation.
\end{abstract}

Electron spins in semiconductors ${ }^{1 / 5}$ are one promising path to quantum computing because of their scalability and long coherence times ${ }^{6}{ }^{8}$. Single qubit gate fidelities exceed $99.9 \%$ in single electron spin qubits $\frac{9}{9}$ and $99 \%$ in singlet-triplet(S-T) qubits $\frac{10}{10}$ S-T qubits 1113 have recently demonstrated two qubit gate fidelities of $90 \%$ by using large magnetic field gradients $\$ 10, \Delta B_{z}$, to diminish the effects of charge noise ${ }^{14}$ and increase coherence times. However, in the presence of $\Delta B_{z}>400 \mathrm{MHz}$ relaxation through coupling to other states reduces readout visibility to almost zerc 15 .

Here we report a new readout scheme that provides readout contrast at large gradients and demonstrate that it has superior performance to previously published methods $\frac{11116 \mid 17}{}$ for $\Delta B_{z}>500 \mathrm{MHz}$ and is faster than other methods that require tunneling processes slow enough to observe charge transitions ${ }^{18 / 21}$. This method is robust up to at least $\Delta B_{z}=900 \mathrm{MHz}$, the largest magnetic field we could generate, and should continue to function in much larger $\Delta B_{z}$. S-T qubits have previously been read out by mapping the qubit states on different charge configurations ${ }^{11}$. However, large gradients enable transitions between the qubit states during measurement, leaving both in the same charge configuration and diminishing contrast. Our technique adds a step before measurement that shelves the qubit states into alternate spin states that do not have relaxation pathways enabled by $\Delta B_{z}$, restoring the ability to map each spin state onto a distinct charge configuration. This method relies on a spin-dependent tunneling between the qubit and the surrounding two dimensional electron gas (2DEG).

To optimize this process, we have measured the visibility of our readout as a function of $\Delta B_{z}$, the voltage applied during shelving and its duration, and magnetic field, $B$. We have also developed a simple model for this readout and used it to simulate our experiments, finding strong agreement with the data. The model we introduce is applicable to other varieties of spin qubits, in-
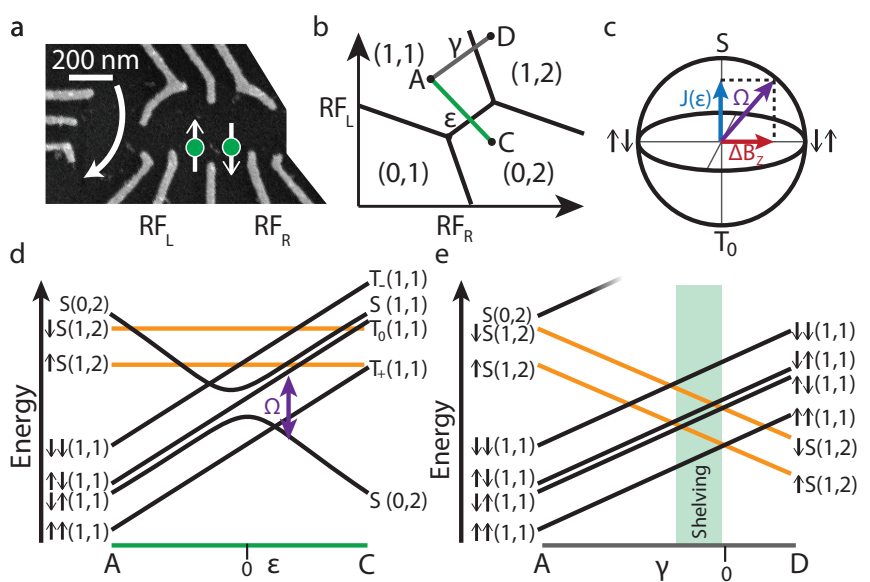

FIG. 1. (a) SEM image of the device. Electron positions are approximated with green circles. The sensor quantum dot is shown with a white arrow. (b) Charge stability diagram of the qubit. In the experiment voltages are either applied equally, $\gamma$, or oppositely, $\epsilon$, to the RF gates. (c) Bloch sphere of the qubit showing the eigenstates of J, $\Delta B_{z}$ and total splitting $\Omega$. (d) The energies of relevant states along the $\epsilon$ curve in b. (e) The energies of relevant states along the $\gamma$ curve in b. In both (d) and (e) black and orange curves represent the energies of two and three electron states respectively.

cluding single spin $18 \mid 19$, hybrid qubit ${ }^{20}$, and donor based $\mathrm{S}-\mathrm{T}$ qubit 21 and latched readout methods $22 \mid 23$ that also rely on tunneling between the qubit and a Fermi sea. This readout technique is general to many host materials, and sources of $\Delta B_{z}$ and to schemes that use S-T readout for single spin qubits 24 .

We study S-T qubits formed from two electrons trapped in an electrostatic gate defined double quantum dot in the 2DEG of GaAs shown in Figure 1a. We use the pair of numbers $(\mathrm{L}, \mathrm{R})$ to represent the number of electrons in the left and right dots respectively. The logical 
subspace for the qubit is made up of the singlet, $|S\rangle=$ $(|\uparrow \downarrow\rangle-|\downarrow \uparrow\rangle) / \sqrt{2}$, and triplet, $\left|T_{0}\right\rangle=(|\uparrow \downarrow\rangle+|\downarrow \uparrow\rangle) / \sqrt{2}$, states where the arrows represent the electron spin in the left and right dot respectively. The Hamiltonian for this system is given by $H=\Delta B_{z} \sigma_{x}+J(\epsilon) \sigma_{z} 11$. The exchange interaction, $J(\epsilon)$, splits $\mathrm{S}$ from $\mathrm{T}_{0}$ and is controlled by the detuning, $\epsilon$, and the energy splitting between $\uparrow \downarrow$ and $\downarrow \uparrow$ is controlled by $\Delta B_{z}$. We call the magnitude of the Hamiltonian $\Omega(\epsilon)=\sqrt{\Delta B_{z}^{2}+J(\epsilon)^{2}}$, as shown in Figure 1c,d. We note that the nature of the qubits ground (excited) state changes from being $S\left(T_{0}\right)$ in $(0,2)$ to $\uparrow \downarrow(\downarrow \uparrow)$ in $(1,1)$.

For all experiments in this work, $\Delta B_{z}$ is produced by the hyperfine interaction with the nuclei, which is controlled through dynamic nuclear polarization (DNP) ${ }^{25}$ applied prior to every experimental run. The qubit is manipulated by applying voltage pulses to the gates labeled $\mathrm{RF}_{L}$ and $\mathrm{RF}_{R}$ in Figure 1a. The total number of electrons in the double dot is controlled by $\gamma=$ $\left(\mathrm{RF}_{L}+\mathrm{RF}_{R}\right) / 2$ and the distribution of these between the right and left dot is controlled by $\epsilon=\mathrm{RF}_{L}-\mathrm{RF}_{R}$, shown in Figure 1b. We define $\gamma=0$ to be the transition from the $(1,1)$ to the $(1,2)$ region, as shown in Figure 1e. The qubit's charge state is measured using an additional neighboring quantum dot $\frac{26}{}$.

We manipulate our qubits deep at Position A, shown in Figure 2a, where the two spins are well isolated so that the ground state is $\downarrow \uparrow(1,1)$ and the excited state is $\uparrow \downarrow(1,1)$. In previous work $\mathrm{S}-\mathrm{T}$ qubits were read out through spin blockade by adiabatically ramping the qubit from deep in $(1,1)$ to the measurement point in the $(0,2)$ region. This point is chosen so that $\mathrm{S}$ is in $(0,2)$ but $\mathrm{T}_{0}$ is spin blockaded to remain in $(1,1)$ because excited energy levels of the quantum dot are energetically inaccessible. This readout process maps $\downarrow \uparrow(1,1)$ to $\mathrm{S}(0,2)$ and $\uparrow \downarrow(1,1)$ to $\mathrm{T}_{0}(1,1)$ so that the distinct charge configurations can be used to measure the qubit's spin state. However, this style of readout is vulnerable because at the measurement point $\Delta B_{z}$ mixes $\mathrm{T}_{0}(1,1)$ with the excited $\mathrm{S}(1,1)$ state, which decays to $\mathrm{S}(0,2)$ on time scales much shorter than the several microsecond measurement time ${ }^{15}$. When this transition occurs, there is no readout contrast because both qubit states have the same charge configuration. The rate of transition from $\mathrm{T}_{0}(1,1)$ to the excited $\mathrm{S}(1,1)$ state increases with $\Delta B_{z}$, meaning that this method has a measurement fidelity that decreases with increasing $\Delta B_{z}$.

To overcome readout failure at large $\Delta B_{z}$ we developed a new readout technique that shelves the qubit states into readout states which do not have relaxation pathways enabled by $\Delta B_{z}$. This new method maps $\downarrow \uparrow(1,1)$ to $\mathrm{S}(0,2)$ and $\uparrow \downarrow(1,1)$ to $\mathrm{T}_{+}(1,1)$. For the remainder of the work, we will refer to this as the the $\mathrm{T}_{+}$readout method. We achieve the desired mapping by using tunneling between the right quantum dot and the 2DEG to change the qubit's spin state. The qubit is tuned so that the left dot is isolated from the lead and the other dot. The shelving process is shown in Figure 2a-c and begins

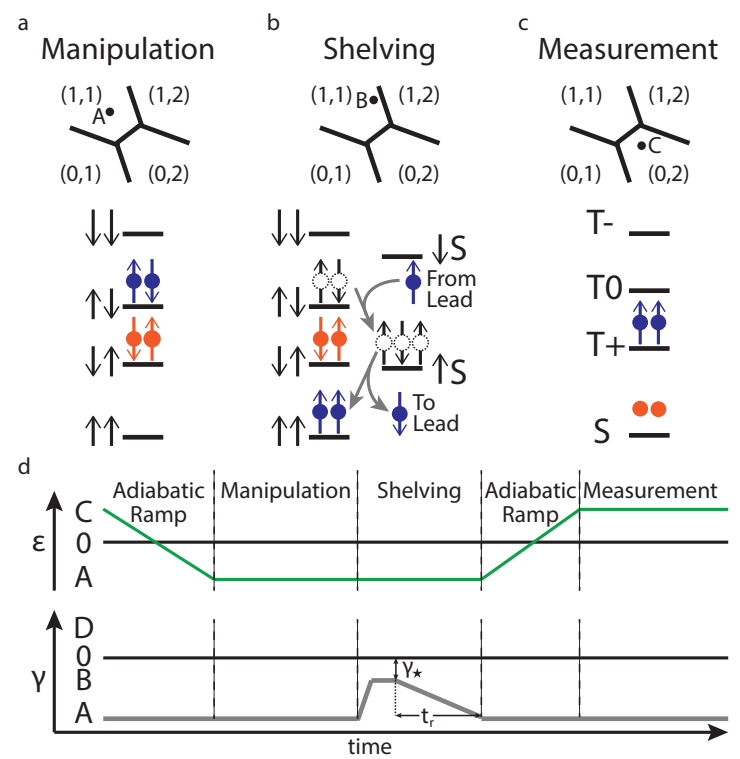

FIG. 2. (a-c) Position in the charge stability diagram and occupation of quantum dot states after (a)manipulation, (b) shelving, and (c) measurement. For (a-c) the qubit's excited state is shown in blue while the qubit ground state is shown in red. (a) After manipulation, the qubit is in its logical subspace, $\uparrow \downarrow$ and $\downarrow \uparrow$. (b) Grey arrows represent the transitions required for shelving to occur. Filled circles show states that are occupied at the end of process while dotted circles show states that are empty. (c) State occupation at the measurement position. The $\mathrm{T}_{+}$and $\mathrm{S}$ states cannot be mixed by $\Delta B_{z}$. (d) Pulse sequence. Values of $\epsilon$ and $\gamma$ during different steps of qubit operation. The shelving position, set by $\gamma_{\star}$ and ramp time, $\mathrm{t}_{r}$, to $(1,1)$ are optimized in Figure 4.

deep in $(1,1)$, at Point A. After manipulation, the qubit is brought to Point B, where $\gamma=\gamma_{\star}$, which is chosen so the required transitions are energetically favorable, as shown in Figure 2b. At this point, electrons can only tunnel in and out of the right dot, enabling the transition from $\uparrow \downarrow(1,1)$ to $\uparrow S(1,2)$ by a spin $\uparrow$ electron tunneling in. The transition from $\downarrow \uparrow(1,1)$ to $\uparrow \mathrm{S}(1,2)$ is blocked because there is no mechanism to change the spin in the left dot. $\uparrow \mathrm{S}(1,2)$ decays to $\uparrow \uparrow(1,1)$ by a spin $\downarrow$ electron tunneling from the right dot to the lead. After allowing the qubit to fully transition, the voltages are adiabatically changed back to Point A over a time $t_{r}$ and then brought to Point $\mathrm{C}$, the same measurement point as in the spin blockade method. The charge state is then measured with $\mathrm{S}(0,2)$ corresponding to the ground state, $\downarrow \uparrow(1,1)$, and $\mathrm{T}_{+}(1,1)$ corresponding to the excited state, $\uparrow \downarrow(1,1)$.

This technique also enables us to measure the direction of $\Delta B_{z}$. We have described this mechanism assuming a specific directionality for $\Delta B_{z}$ but it functions with the opposite orientation as well. Flipping the direction of $\Delta B_{z}$ causes $\uparrow \downarrow(1,1)$ to be the ground state and $\downarrow \uparrow(1,1)$ to be the excited state. This readout still maps $\uparrow \downarrow(1,1)$ to $\mathrm{T}_{+}(1,1)$ while $\downarrow \uparrow(1,1)$ is initially mapped to $\mathrm{T}_{0}(1,1)$ and quickly decays to the $\mathrm{S}(0,2)$ charge state through 
the mechanism previously described. This inverts the charge signal we measure from the qubit ground state, allowing for a direct measurement of the direction of $\Delta B_{z}$. In these experiments, $\Delta B_{z}$ is oriented as in the second regime because DNP is more effective when pumping with $\mathrm{T}_{+}$than $\mathrm{S}$, as detailed in the Supplementary Materials 27 .

These readout techniques are sufficient for full qubit state tomography because we are able to pair them with high fidelity single qubit gates. We can measure along any axis by performing the proper rotations so that the states along the desired axis are mapped onto $\uparrow \downarrow(1,1)$ and $\downarrow \uparrow(1,1)$.

We have constructed a simple model for the $\mathrm{T}_{+}$ method that captures the experimental trends that we observe and offers intuition for this technique's behavior. To determine the equilibrium populations of all the different quantum dot states, we have calculated the transition rates between all pairs of states using Fermi's golden rule to compute the tunneling rates of electrons between the qubit and the 2DEG. We find the following transition rates, $\Gamma_{i j}$ between the $(1,1)$ states, $i$, and the $(1,2)$ states, $j$, and the reverse, $\Gamma_{j i}$ :

$$
\begin{aligned}
\Gamma_{i j} & =\frac{2 \pi}{\hbar}|\langle j|\tau| i\rangle|^{2} f\left(\Delta E_{i j}, T, \mu\right) \rho_{f} \\
\Gamma_{j i} & =\frac{2 \pi}{\hbar}|\langle i|\tau| j\rangle|^{2}\left(1-f\left(-\Delta E_{j i}, T, \mu\right)\right) \rho_{f}
\end{aligned}
$$

Here $\hbar$ is the reduced Planck constant, $\tau$ is the tunneling term between the right quantum dot and 2DEG, $\mathrm{f}$ is the Fermi-Dirac distribution, $\Delta E_{i j}=E_{j}-E_{i}$ is energy difference between $i$ and $j, T$ is the electron temperature, and $\mu$ and $\rho_{f}$ are the chemical potential and density of states of the 2DEG. $\Delta \mathrm{E}_{i j}$ is controlled by $\epsilon, \gamma, \Delta B_{z}$, and $B$. Transitioning between states with different numbers of electrons requires an electron tunneling to or from the lead with an energy that compensates for any change to the qubit's energy. The Fermi-Dirac distribution dictates the number of electrons and holes available for $\Gamma_{i j}$ and $\Gamma_{j i}$ respectively, which governs the rates. This means that the transition rates from states with lower energy to higher energy are suppressed because they require an excited electron or hole to donate the energy difference. We note that many rates are 0 due to spin conservation, suppressing transitions between states with incompatible spin configurations. We use these rates to simulate the transitions that occur during $\mathrm{T}_{+}$readout so that we can perform simulations while varying the same parameters as we do experimentally. Details are included in the Supplementary Materials 27 .

We determined the contrast of the readout methods that we tested by finding the measurement fidelity ${ }^{26}$ for the ground, $\mathrm{F}_{G}$ and excited states, $\mathrm{F}_{E}$, as detailed in the Supplementary Materials ${ }^{27}$. We used these quantities to calculate the visibility, given by $\mathrm{F}_{G}+\mathrm{F}_{E}-1$. In Figure 3 a we present the measured visibility of spin blockade and $\mathrm{T}_{+}$readout techniques as a function of $\Delta B_{z}$ with an
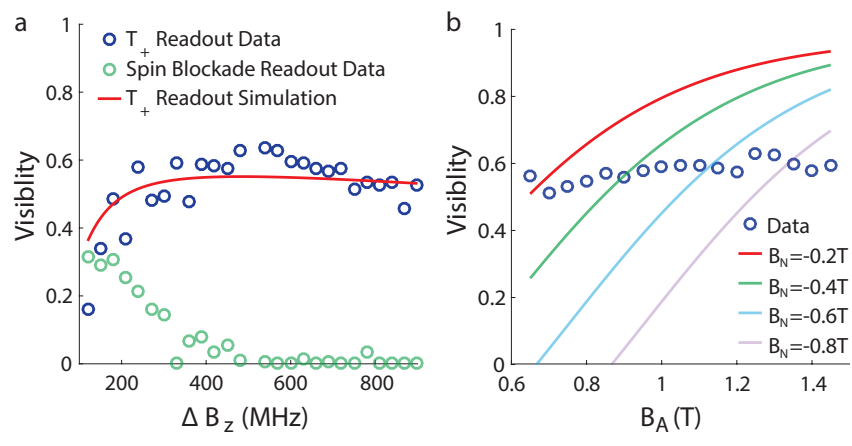

FIG. 3. (a) Measurements of the visibility of the spin blockade and $\mathrm{T}_{+}$readout methods as a function of $\Delta B_{z}$. Red curve is a simulation of the $T_{+}$method. The visibility of the $T_{+}$ method is superior at large $\Delta B_{z}$. (b) Measurement and simulations with varied $B_{N}$ of the visibility of the $\mathrm{T}_{+}$method as a function of $B_{A}$. The data does not follow one simulation curve, suggesting that the $B_{N}$ produced by DNPS is a function of $B_{A}$.

applied field $\mathrm{B}_{A}=0.7 \mathrm{~T}$. In Figure $3 \mathrm{a}$ we also present a simulation for the visibility of the $\mathrm{T}_{+}$readout and note the agreement with the data.

We see that the spin blockade readout visibility decreases very quickly with increasing $\Delta B_{z}$ as we expect from the increasing decay rate from $\mathrm{T}_{0}(1,1)$ to $\mathrm{S}(0,2)$ at the measurement point. The $\mathrm{T}_{+}$readout is poor at small $\Delta B_{z}$ because $\mathrm{J}(\epsilon)$ is comparable to $\Delta B_{z}$ which gives both qubit states the ability to decay to $\uparrow \mathrm{S}(1,2)$. However, the $\mathrm{T}_{+}$method has large visibility for $\Delta B_{z}>200 \mathrm{MHz}$. We note also the slow fall off of visibility for $\Delta B_{z}>500$ MHz. This is due to $\Delta B_{z}$ decreasing the energy splitting between the $\uparrow \downarrow$ state and the $\uparrow \uparrow$ state, decreasing the thermodynamic equilibrium occupation of $\uparrow \uparrow$, as can be seen from the energies given in the Supplementary Materials27. Flipping the direction of $\Delta B_{z}$ would give a weak improvement instead because $\Delta B_{z}$ would increase the energy difference between $\uparrow \uparrow$ and $\uparrow \downarrow$ rather than decrease it. We compare the performance of the $T_{+}$and another previously published readout method ${ }^{17}$ as a function of $\Delta B_{z}$ in the Supplementary Materials 27 .

In Figure $3 \mathrm{~b}$ we present the data for the $\mathrm{T}_{+}$readout method visibility versus the applied magnetic field, $B_{A}$. We find only a weak dependence on the $B_{A}$ while the model predicts a sharp increase. Past measurements have shown that DNP pumps both the difference field, $\Delta B_{z}$, and sum field, $B_{N}$ experienced quantum dots due to the polarized nuclei. The magnetic field experienced by the qubit is $B=B_{A}+B_{N}$. Pumping with $\mathrm{T}_{+}$states flips nuclei such that $B_{N}<0$ while pumping with $\mathrm{S}$ states yields $B_{N}>0$. While measuring the data presented in Figure $3 \mathrm{~b}$, we observed increasing DNP times required for a given value of $\Delta B_{z}$ to the extent that it took 10 times longer to stabilize $\Delta B_{Z}$ at $B_{A}=1.4 \mathrm{~T}$ than at $B_{A}=0.7$ $\mathrm{T}$. This suggests that nuclei are flipped more symmetrically between the dots with increasing $B_{A}$, yielding larger magnitude $B_{N}$, because DNP is less efficient at pumping 

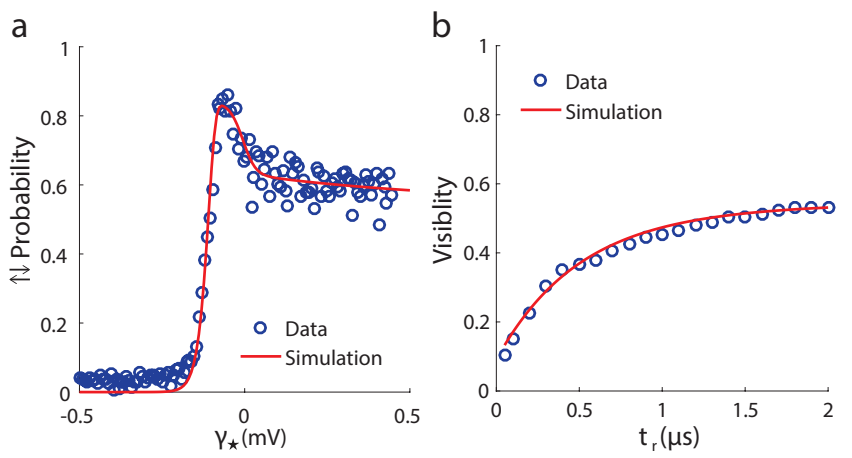

FIG. 4. (a) Measurements and simulation for the probability that the $\uparrow \downarrow$ state correctly transitions as a function of $\gamma_{\star}$. The peak occurs where the required transitions are energetically favorable while still keeping undesirable transitions unfavorable. (b) Measurement and simulation of the visibility of the $\mathrm{T}_{+}$as a function of $\mathrm{t}_{r}$. Longer times allow the qubit to more completely transition to the desired end state.

$\Delta B_{z}$. In Figure $3 \mathrm{~b}$ we plot simulations at several different $B_{N}$ and see that the data transition between curves with increasingly negative $B_{N}$, consistent with DNP becoming less effective at generating $\Delta B_{z}$ at larger $B_{A}$. The magnetic field dependence of DNP pumping rates of $\Delta B_{z}$ and $B_{N}$ is a subject of current investigation.

The fidelity of the $\mathrm{T}_{+}$readout method depends strongly on the readout position because the technique relies on the desired transitions being energetically favorable while the undesired transitions remain unfavorable. The energy spectrum of available states as a function of $\gamma$ is shown in Figure 1e. We select the optimal readout position by repeatedly preparing $\uparrow \downarrow(1,1)$ and immediately attempting to measure at readout positions with different $\gamma_{\star}$, as shown in Figure 2d. We plot data and a simulation of the probability that the measurement correctly identified the $\uparrow \downarrow$ state in Figure 4a.

When $\gamma_{\star} \ll 0$ the $\uparrow \mathrm{S}(1,2)$ state has far more energy than $\uparrow \downarrow(1,1)$, preventing the first transition required for $\mathrm{T}_{+}$readout. As $\gamma_{\star}$ approaches zero $\uparrow \mathrm{S}(1,2)$ comes into resonance with $\uparrow \downarrow(1,1)$ and we see a dramatic upturn in the probability of transitioning because there are thermally excited electrons that allow for the first transition. When $\gamma_{\star}>0$ the probability drops again because the desired end state, $\uparrow \uparrow(1,1)$ is not the lowest in energy during the readout process so it is not the most thermodynamically populated. All other measurements in this paper were performed at the optimal measured readout position. Our simulation is only for the shelving process and does not incorporate errors due to incorrectly reading out the charge state. Incorrect charge readout prevents the $\uparrow \downarrow$ probability from being measured at exactly 0 for $\gamma_{\star} \ll 0$ as the simulation would predict.

To optimize the $T_{+}$readout, we also investigated the dependence of the visibility on $t_{r}$. Our simulations and experiments showed little dependence on how quickly $\gamma$ was increased to ramp the voltages from Point A to Point
$\mathrm{B}$, where $\gamma=\gamma_{\star}$, but a strong dependence on the time, $\mathrm{t}_{r}$, over which $\gamma$ was varied to change the voltages back from Point B back to Point A. We present measurements and simulations for the visibility as a function of $t_{r}$ in Figure 4b. The visibility sharply improves with increasing $t_{r}$ because the qubit has time to equilibrate as $\gamma$ is varied resulting in a higher occupation of $\mathrm{T}_{+}$. At very short times, $(\uparrow, S)$ is rapidly raised above $\uparrow \downarrow$ state, allowing for undesirable transitions and reducing visibility.

The maximum visibility that we observe is approximately 0.6 , corresponding to an average readout fidelity of $80 \%$. This is limited by the equilibrium thermodynamic occupation of the states that the qubit transitions through during the shelving process. This thermodynamic limit can be improved by decreasing the electron temperature or by using $\Delta B_{z}$ and $B$ to increase the energy splittings between the states used for shelving. As mentioned above, the direction of $\Delta B_{z}$ can be chosen so that it increases the relevant splittings. While the direction of $\Delta B_{z}$ in these experiments was governed by using DNP and decreased the relevant splittings, the direction is more flexible when generated by a micromagnet $\left[\begin{array}{l}3128 \\ 30\end{array}\right.$ so that visibility can instead be enhanced. Another benefit of using a micromagnet is that $B_{N}$ will remain fixed, so that we have direct control of $B$ through $B_{A}$. We expect to observe the behavior predicted by the simulations in Figure 3b, allowing this method to achieve visibilities above $90 \%$ by increasing $B_{A}$.

We demonstrated that the $\mathrm{T}_{+}$readout method allows for measurements with large $\Delta B_{z}$, a regime that was previously inaccessible due to low readout visibility. We have also demonstrated that calibrating $t_{r}$ and $\gamma_{\star}$ is critical to optimizing the visibility. Additionally, we have identified that using an external source of $\Delta B_{z}$, such as a micromagnet, should enable higher fidelity readout by the application of larger $B_{A}$ and prudently selecting the direction of $\Delta B_{z}$. We expect that these changes should enable visibilities in line with other high quality qubit readouts. The $\mathrm{T}+$ readout technique is also applicable to scalable architectures that map a single spin qubit onto S-T states for readout ${ }^{24}$.

The concept of using a shelving step before measurement is relevant to any system where readout is limited by decay processes during measurement. We have demonstrated that visibilities can be increased by transferring the qubit into states that are immune to the decay pathway before measurement. We have also developed a method for simulating processes that rely on spin dependent tunneling between a quantum dot and a reservoir. This can be used to optimize the initialization and readout in a wide variety of qubits because they rely on these tunneling processes. Our demonstration of using experiments and simulations to develop the $\mathrm{T}_{+}$readout method can serve as a guide for other researchers who need to develop readout schemes tailored to their specific experimental requirements. 


\section{ACKNOWLEDGMENTS}

Work at Harvard was funded by Army Research Office grants W911NF-15-1-0203 and W911NF-17-1-024. Samples were fabricated at the Harvard University Center for Nanoscale Systems (CNS), a member of the National Nanotechnology Infrastructure Network (NNIN), which is supported by the National Science Foundation under NSF award No. ECS0335765. Work at Purdue was sup- ported by the Department of Energy, Office of Basic Energy Sciences, under Award number DE-SC0006671. Additional support for the MBE growth facility from the W. M. Keck Foundation and Nokia Bell Laboratories is gratefully acknowledged. S.P.H. was supported by the Department of Defense through the National Defense Science Engineering Graduate Fellowship Program. L.A.O. was supported by the Army Research Office through the Quantum Computing Graduate Research Fellowship Program.
1 D. Loss and D. P. DiVincenzo, Phys. Rev. A. 57, 120 (1998).

2 F. H. L. Koppens, C. Buizert, K. J. Tielrooij, I. T. Vink, K. C. Nowack, T. Meunier, L. P. Kouwenhoven, and L. M. K. Vandersypen, Nature 442, 766 (2006).

3 M. Pioro-Ladriere, T. Obata, Y. Tokura, Y. S. Shin, T. Kubo, K. Yoshida, T. Taniyama, and S. Tarucha, Nature Physics 4, 776 (2008).

${ }^{4}$ D. Kim, Z. Shi, C. B. Simmons, D. R. Ward, J. R. Prance, T. S. Koh, J. K. Gamble, D. E. Savage, M. G. Lagally, M. Friesen, S. N. Coppersmith, and M. A. Eriksson, Nature 511, 70 (2014).

${ }^{5}$ K. Eng, T. D. Ladd, A. Smith, M. G. Borselli, A. A. Kiselev, B. H. Fong, K. S. Holabird, T. M. Hazard, B. Huang, P. W. Deelman, I. Milosavljevic, A. E. Schmitz, R. S. Ross, M. F. Gyure, and A. T. Hunter, Science Advances 1, e1500214 (2015).

${ }^{6}$ M. Veldhorst, J. C. C. Hwang, C. H. Yang, A. W. Leenstra, B. de Ronde, J. P. Dehollain, J. T. Muhonen, F. E. Hudson, K. M. Itoh, A. Morello, and A. S. Dzurak, Nature Nanotechnology 9, 981985 (2014).

7 J. T. Muhonen, J. P. Dehollain, A. Laucht, F. E. Hudson, R. Kalra, T. Sekiguchi, K. M. Itoh, D. N. Jamieson, J. C. McCallum, A. S. Dzurak, and A. Morello, Nature Nanotechnology 9, 986 (2014).

8 K. Saeedi, S. Simmons, J. Z. Salvail, P. Dluhy, H. Riemann, N. V. Abrosimov, P. Becker, H.-J. Pohl, J. L. Morton, and M. L. W. Thewalt, Science 342, 830 (2013).

9 J. Yoneda, K. Takeda, T. Otsuka, T. Nakajima, M. R. Delbecq, G. Allison, T. Honda, T. Kodera, S. Oda, Y. Hoshi, N. Usami, K. M. Itoh, and S. Tarucha, arxiv (2018).

10 J. M. Nichol, L. A. Orona, S. P. Harvey, S. Fallahi, G. C. Gardner, M. J. Manfra, and A. Yacoby, Nature Quantum Information 3 (2017).

11 J. R. Petta, A. Johnson, J. M. Taylor, E. A. Laird, A. Yacoby, M. D. Lukin, C. Marcus, M.P.Hanson, and A.C.Gossard, Science 309, 2180 (2005).

12 S. Folleti, H. Bluhm, D. Mahalu, V. Umansky, and A. Yacoby, Nature Physics 5, 903 (2009).

13 M. D. Shulman, O. E. Dial, S. P. Harvey, H. Bluhm, V. Umansky, and A. Yacoby, Science 336, 202 (2012).

14 O. E. Dial, M. D. Shulman, S. P. Harvey, H. Bluhm, V. Umansky, and A. Yacoby, Phys. Rev. Lett 110, 146804 (2013).

15 C. Barthel, J. Medford, H. Bluhm, A. Yacoby, C. M. Marcus, M. P. Hanson, and A. C. Gossard, Phys. Rev. B 85, 035306 (2012).

${ }^{16}$ K. D. Petersson, J. R. Petta, H. Lu, and A. C. Gossard, Phys. Rev. Lett 105, 246804 (2010).
17 S. A. Studenikin, J. Thorgrimson, G. C. Aers, A. Kam, P. Sawadski, Z. R. Wasilewski, A. Bogan, and A. S. Sachrajda, Appl. Phys. Lett 101, 233101 (2012).

18 J. M. Elzerman, R. Hanson, L. H. W. van Beveren, B. Witkamp, L. M. K. Vandersypen, and L. P. Kouwenhoven, Nature (2004).

19 A. Morello, J. J. Pla, F. A. Zwanenburg, K. W. Chan, K. Y. Tan, H. Huebl, M. Mttnen, C. D. Nugroho, C. Yang, J. A. van Donkelaar, A. D. C. Alves, D. N. Jamieson, C. C. Escott, L. C. L. Hollenberg, R. G. Clark, and A. S. Dzurak, Nature 467, 687 (2010).

20 D. Kim, D. R. Ward, C. B. Simmons, D. E. Savage, M. G. Lagally, M. Friesen, S. N. Coppersmith, and M. A. Eriksson, Nature Quantum Information 1, 70.

${ }^{21}$ M. A. Broome, T. F. Watson, D. Keith, S. K. Gorman, M. G. House, J. G. Keizer, S. J. Hile, W. Baker, and M. Y. Simmons, Phys. Rev. Letters 119, 046802 (2017).

${ }^{22}$ P. Harvey-Collard, B. DAnjou, M. Rudolph, N. T. Jacobson, J. Dominguez, G. A. T. Eyck, J. R. Wendt, T. Pluym, M. P. Lilly, W. A. Coish, M. Pioro-Ladrire, and M. S. Carroll, arxiv (2017).

23 T. Nakajima, M. R. Delbecq, T. Otsuka, P. Stano, S. Amaha, J. Yoneda, A. Noiri, K. Kawasaki, K. Takeda, G. Allison, A. Ludwig, A. D. Wieck, D. Loss, and S. Tarucha, arxiv (2017).

24 M. A. Fogarty, K. Chan, B. Hensen, W. Huang, T. Tanttu, C. Yang, A. Laucht, M. Veldhorst, F. Hudson, K. Itoh, D. Culcer, A. Morello, and A. Dzurak, arxiv (2017).

25 H. Bluhm, S. Foletti, D. Mahalu, V. Umansky, and A. Yacoby, Phys. Rev. Lett 105, 216803 (2010).

26 C. Barthel, D. J. Reilly, C. M. Marcus, M. P. Hanson, and A. C. Gossard, Phys. Rev. Letters 103, 160503 (2009).

27 See Supplemental Material at [url will be inserted by publisher] for further experimental and theoretical details.

${ }^{28}$ K. Takeda, J. Kamioka, T. Otsuka, J. Yoneda, T. Nakajima, M. R. Delbecq, S. Amaha, G. Allison, T. Kodera, S. Oda, and S. Tarucha, Science Advances 2, e1600694 (2016).

29 X. Wu, D. R. Ward, J. R. Prance, D. Kim, J. K. Gamble, R. T. Mohra, Z. Shia, D. E. Savage, M. G. Lagally, M. Friesen, S. N. Coppersmith, and M. A. Eriksson, PNAS 111, 1193811942 (2014).

30 E. Kawakami, P. Scarlino, D. R. Ward, F. R. Braakman, D. E. Savage, M. G. Lagally, M. Friesen, S. N. Coppersmith, M. A. Eriksson, and L. M. K. Vandersypen, Nature Nanotechnology 9, 666 (2014).

31 J. Emerson, R. Alicki, and K. Zyckowski, arXiv (2005).

32 C. Danker, R. Cleve, J. Emerson, and E. Livine, arXiv (2006). 
${ }^{33}$ B. Levi, C. C. Lopez, J. Emerson, and D. G. Cory, arXiv

(2006).

34 J. Bardeen, Phys. Rev. Lett 6, 57 (1961). 